REVISTA CIENCIAS BIOMÉDICAS

ARTÍCULOS ORIGINALES

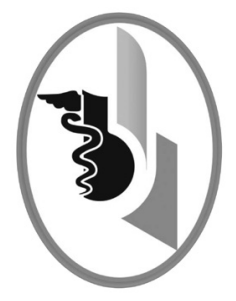

\title{
CARACTERIZACIÓN DE LAS INFECCIONES RESPIRATORIAS ASOCIADAS A VENTILACIÓN MECÁNICA EN UNA UNIDAD DE CUIDADOS INTENSIVOS
}

\author{
CHARACTERIZATION OF THE VENTILATOR-ASSOCIATED \\ RESPIRATORY INFECTIONS IN AN INTENSIVE CARE UNIT
}

Cogollo-González Marysabel ${ }^{1}$

Julio-Narváez Luis Carlos²

Alvarado-Cueto Daniel Enrique ${ }^{2}$

Correspondencia: marysabel088@hotmail.com

Recibido para evaluación: julio - 15-2014. Aceptado para publicación: abril - 26-2015.

\section{RESUMEN}

Introducción: las infecciones respiratorias asociadas a ventilación mecánica (IRAV), sean neumonía o traqueobronquitis, son importantes complicaciones en UCI.

Objetivo: caracterizar los aspectos epidemiológicos, clínicos y microbiológicos de IRAV. Materiales y método: estudio retrospectivo de todos los pacientes admitidos a la UCI Médico Quirúrgica de la Clínica San Juan de Dios, durante el periodo de un año, con diagnóstico de traqueobronquitis asociada a ventilación mecánica (TAV) o neumonía asociada a ventilación mecánica (NAV), con cultivos de aspirado de secreción traqueal informados como positivos.

Resultados: se presentaron 22 casos de IRAV. La presencia de IRAV fue del $11.9 \%$, de TAV $6.8 \%$ y de NAV $5.6 \%$ de los 176 pacientes que requirieron ventilación mecánica por más de dos días. La mediana de edad para NAV fue de $76 \pm 16$ años, mientras que para la TAV de $68 \pm 18$ años. Los principales diagnósticos de ingreso de los pacientes con IRAV fueron neurológicos $(40.9 \%)$ y pulmonares $(31.8 \%)$. Predominaron los gérmenes gram negativos $(95.5 \%)$ y los microrganismos multirresistentes en ambos tipos de infecciones (59.1\%). Hubo alta frecuencia de uso de antibioticoterapia previa al diagnóstico (95.5\%), traqueostomía (81.8\%) y transfusiones de hemoderivados (50\%). La mortalidad en UCI fue $25 \%$ para TAV y $40 \%$ para NAV, mientras que la mortalidad hospitalaria de IRAV fue $50 \%$.

Conclusión: existe alta mortalidad, presencia de microorganismos gram negativos involucrados y elevada multirresistencia antibiótica en ambas formas de IRAV. Se deben implementar medidas encaminadas a la prevención y al adecuado manejo de estas infecciones en UCI. Rev.cienc.biomed. 2015;6(1):38-44

\section{PALABRAS CLAVE}

Dispositivos de protección respiratoria; Respiración artificial; Cuidados intensivos; Neumonía bacteriana.

1 Médico General. Clínica Madre Bernarda. Cartagena. Colombia.

2 Médico. Internista. Clínica San Juan de Dios. Clínica Madre Bernarda. Cartagena. Colombia. Aspirante a Magíster Cuidado Crítico e Intensivo. Universidad San Jorge. España. 


\section{SUMMARY}

Introduction: the ventilator-associated respiratory infections (VARI), pneumonia or tracheobronchitis, are important complications in intensive care units (ICU).

Objective: To characterize the epidemiological, clinical and microbiological aspects of VARI.

Methods: retrospective study of all patients who were admitted to the ICU of the Clínica San Juan de Dios, during the period of one year, with diagnosis of Ventilator-Associated tracheobronchitis (VAT) or Ventilator-associated pneumonia (VAP), with cultures of endotraqueal secretion informed as positive.

Results: 22 cases of VARI were presented. The presence of VARI was $11.9 \%$, VAT $6.8 \%$ and VAP $5.6 \%$ of the 176 patients who required mechanical ventilation for more than two days. The median of age for VAP was $76 \pm 16$ years, while for VAT of $68 \pm 18$ years. The main diagnosis of admission of the patients with VARI were neurological $(40.9 \%)$ and pulmonary $(31.8 \%)$. The gram-negative $(95.5 \%)$ and multidrug-resistant microorganisms of both infections $(59.1 \%)$ predominated. Also, there was high frequency of use of antibioticotherapy previous to the diagnosis (95.5\%), tracheostomy $(81.8 \%)$ and transfusion of hemoderivative (50\%). The mortality in ICU was $25 \%$ for VAT and $40 \%$ por VAP, while the in-hospital mortality of VARI was $50 \%$.

Conclusion: there is high mortality, presence of gram-negative microorganisms involved and high multidrug resistance in both forms of VARI. Measures oriented to the prevention and management of these infections in ICU must be implemented. Rev. cienc.biomed. $2015 ; 6(1): 38-44$

\section{KEYWORDS}

Respiratory protective devices; Artificial respiration; Intensive care; Bacterial pneumonia.

\section{INTRODUCCIÓN}

Con el fin de restablecer la homeostasis del paciente crítico, en el escenario de la UCI, este se expone a complejos procedimientos y métodos terapéuticos invasivos, como la intubación orotraqueal y el posterior soporte ventilatorio, lo cual brinda al paciente la oportunidad de vida, pero a su vez se convierte en riesgo de padecer infecciones (1).

La neumonía asociada a ventilación mecánica (NAV) es la más estudiada, con una incidencia que varía dependiendo de los criterios de definición aplicados. Presenta gran impacto en desenlace, dado que aumenta el tiempo de estancia hospitalaria, la duración de ventilación mecánica, la mortalidad y los costos; se ha estimado de 40.000 a 57.000 dólares, más alto que en pacientes sin NAV (1-4).

Son pocos los datos sobre la traqueobronquitis asociada a ventilación mecánica (TAV), entidad que también se ha relacionado con incremento en los días de UCI y de necesidad de ventilación mecánica (5).

La región Caribe colombiana carece de suficientes publicaciones acerca de infecciones respiratorias asociadas a ventilación mecá- nica (IRAV). El objetivo del estudio es caracterizar aspectos epidemiológicos, clínicos y microbiológicos de los pacientes que presentan IRAV en la UCI.

\section{MATERIALES Y MÉTODO}

Estudio retrospectivo. Población compuesta por todos los pacientes admitidos, entre 23 de diciembre de 2012 y 23 de diciembre de 2013 a la UCI Medico Quirúrgica de la Clínica San Juan de Dios, Cartagena, Colombia. Los datos se recogieron mediante la revisión de las historias clínicas de los pacientes y la base de datos del servicio de terapia respiratoria de la UCI.

Se incluyeron pacientes mayores de 18 años de edad que requirieron ventilación mecánica (VM) por más de 48 horas. El diagnóstico de las IRAV se basó en lo propuesto por Bouza et al (6). Para la NAV, presencia de nuevo y/o progresivo infiltrado pulmonar en una radiografía de tórax, asociado con al menos dos de los siguientes criterios: [A] Secreción traqueal purulenta. [B] Recuento de leucocitos $>12.000 \circ<4.000 / \mathrm{mm}^{3}$. [C] Temperatura axilar $>38^{\circ} \mathrm{C} o<36^{\circ} \mathrm{C}$. [D] Empeoramiento de la relación presión arterial de oxígeno/fracción inspiratoria de oxígeno ( $\mathrm{PaO} 2 / \mathrm{FiO} 2)$ en las 48 horas previas. 
A su vez la TAV se definió como la ausencia de infiltrados nuevos o progresivos en los campos pulmonares, presencia de secreciones traqueales purulentas, leucocitos $>12.000$ o $<4.000 / \mathrm{mm}^{3} \mathrm{y} / \mathrm{o}$ temperatura axilar $>38^{\circ} \mathrm{C} 0<36^{\circ} \mathrm{C}$. Posteriormente se eligieron los pacientes con cultivos de aspirado traqueal positivos para microrganismos con recuento mayor o igual a 100.000 unidades formadoras de colonias (5).

Se excluyeron aquellos pacientes con diagnóstico previo de NAV o TAV que llegaron remitidos de otra institución.

Se midieron las características epidemiológicas: incidencia, edad y sexo; características clínicas: diagnóstico principal, antecedentes, escala de severidad para Evaluación Fisiológica Aguda y de Salud Crónica (APACHE II), sistema de puntuación para Evaluación de Fallo Orgánico Secuencial (SOFA), necesidad de traqueostomía, tipo de traqueostomía ( $p r e c o z$, si fue realizada entre los primeros siete días después de la intubación orotraqueal o tardía si fue mayor a este tiempo), uso de vasoactivos, antibioticoterapia previa, requerimiento de transfusiones, días de ventilación mecánica, estancia en UCI, tiempo de hospitalización, destete ventilatorio, mortalidad en UCI y hospitalaria; y las características microbiológicas: tipo de infección (temprana si se presentó en los primeros cuatro días después de la intubación orotraqueal y tardía si fue mayor a este tiempo) clase de germen de acuerdo a la coloración gram, tipo de germen encontrado y sensibilidad a fármacos.

Los datos se recogieron y analizaron utilizando el programa Epi Info (Epi Info versión 3.5.1; CDC; Atlanta, EE.UU.). Las variables cualitativas se expresaron en porcentajes, mientras que las cuantitativas continuas se registraron en medidas de tendencia central. El estudio fue aprobado por parte del Comité de Ética de la Clínica San Juan de Dios, Cartagena, Colombia.

\section{RESULTADOS}

Durante el periodo de estudio fueron admitidos 680 pacientes a la UCI de la Clínica San Juan de Dios. Los pacientes que requirieron VM por más de dos días fueron 176 pacientes $(25.8 \%)$, de los cuales $21(11.9 \%)$ cumplieron criterios diagnósticos de IRAV. La presencia de TAV fue del $6.8 \%$ y de NAV del $5.6 \%$ de los pacientes que requirieron VM superior a dos días(Figura $\mathrm{N}^{\circ} 1$ ).

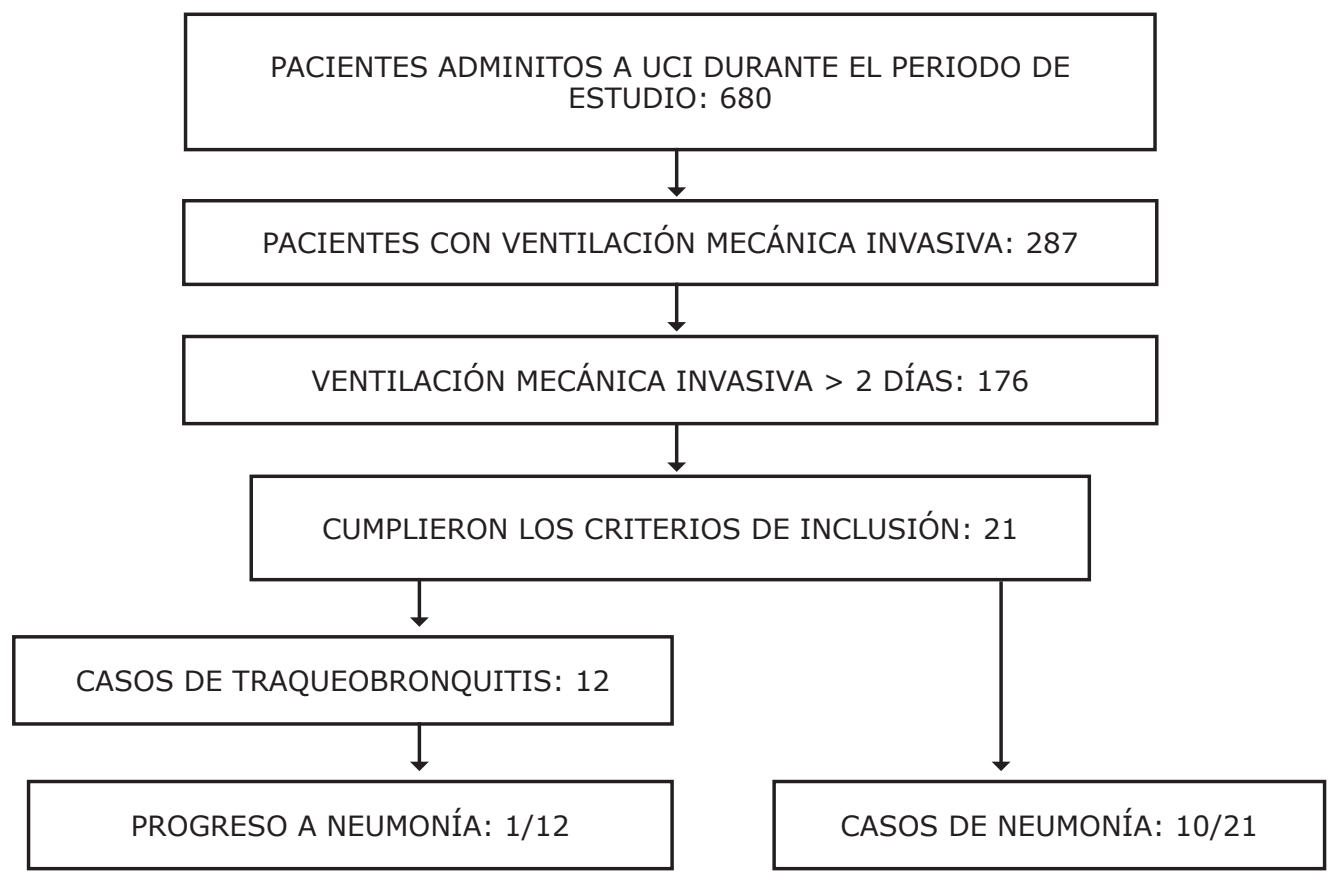

Figura 1. Flujograma de pacientes 
Se observó que el promedio de edad para NAV fue de 76 años con una desviación estándar de 16 años, mientras que para la TAV el promedio fue de 68 años con una desviación estándar de 18 años. La mayoría provenían del área urbana, remitidos del servicio de urgencias, el resto de las características sociodemográficas se observan en la Tabla No 1.

En cuanto a las características clínicas (Tabla No 2) el promedio de puntuación de APACHE II fue de 20 con desviación estándar de 8 . Se observó en ambos tipos de infecciones un tiempo de estancia a UCI y al ventilador mecánico por más de veinte días. El principal diagnóstico fue el neurológico, seguido del pulmonar y el cardiológico. Los principales antecedentes tanto para TAV y NAV fueron hipertensión arterial, enfermedad pulmonar obstructiva crónica (EPOC) y diabetes mellitus.

El tipo de infección predominante fue la tardía. En la mayoría de los pacientes se observó uso de antibioticoterapia previa, transfusiones de hemoderivados y necesidad de traqueostomía. La realización de este procedimiento de forma tardía fue en el $59 \%$ de los pacientes que la requirieron. La mortalidad en UCI fue del $31.8 \%$ (25.0\% para TAV y $40.0 \%$ para NAV), siendo del $50.0 \%$ el egreso hospitalario del total de las IRAV.

Los principales gérmenes observados fueron gram
TABLA No 1.

CARACTERÍSTICAS EPIDEMIOLÓGICAS DE LAS IRAV EN UCI DE LA CLÍNICA SAN JUAN DE DIOS

\begin{tabular}{|l|c|c|c|}
\hline & $\begin{array}{c}\text { TAV } \\
\mathrm{n}=12\end{array}$ & $\begin{array}{c}\text { NAV } \\
\mathrm{n}=10\end{array}$ & $\begin{array}{c}\text { IRAV } \\
\mathrm{n}=22\end{array}$ \\
\hline Edad, $\mathrm{X} \pm \mathrm{DE}$ & $68 \pm 18$ & $76 \pm 16$ & $70 \pm 17$ \\
\hline \multicolumn{4}{|c|}{ Sexo, $\mathrm{n}(\%)$} \\
\hline Femenino & $5(42)$ & $5(50)$ & $10(45)$ \\
\hline Masculino & $7(58)$ & $5(50)$ & $12(55)$ \\
\hline \multicolumn{4}{|c|}{ Procedencia, $\mathrm{n}(\%)$} \\
\hline Urbano & $12(100)$ & $7(70)$ & $19(86)$ \\
\hline Rural & $0(0)$ & $3(30)$ & $3(14)$ \\
\hline
\end{tabular}

\begin{tabular}{|c|c|c|c|c|c|c|}
\hline \multicolumn{7}{|c|}{$\begin{array}{l}\text { TABLA No } 2 . \\
\text { CARACTERÍSTICAS CLÍNICAS DE LAS IRAV EN UCI DE LA } \\
\text { CLÍNICA SAN JUAN DE DIOS }\end{array}$} \\
\hline & \multicolumn{2}{|c|}{$\begin{array}{c}\mathrm{TAV} \\
\mathrm{n}=12\end{array}$} & \multicolumn{2}{|c|}{$\begin{array}{l}\text { NAV } \\
n=10\end{array}$} & \multicolumn{2}{|c|}{$\begin{array}{l}\text { IRAV } \\
n=22\end{array}$} \\
\hline APACHE II $*,+$ & \multicolumn{2}{|c|}{$21 \pm 9$} & \multicolumn{2}{|c|}{$20 \pm 7$} & \multicolumn{2}{|c|}{$20 \pm 8$} \\
\hline SOFA,$+ \neq$ & \multicolumn{2}{|c|}{$5+2$} & \multicolumn{2}{|r|}{$6 \pm 3$} & \multicolumn{2}{|c|}{$5 \pm 2$} \\
\hline $\begin{array}{l}\text { Días de } \\
\text { ventilación } \\
\text { mecánica † }\end{array}$ & \multicolumn{2}{|c|}{$20 \pm 13$} & \multicolumn{2}{|c|}{$22 \pm 12$} & \multicolumn{2}{|c|}{$22 \pm 12$} \\
\hline $\begin{array}{l}\text { Días de estancia } \\
\text { en UCI }+\end{array}$ & \multicolumn{2}{|c|}{$21 \pm 12$} & \multicolumn{2}{|c|}{$26 \pm 11$} & \multicolumn{2}{|c|}{$24 \pm 11$} \\
\hline \multicolumn{7}{|c|}{ Diagnóstico, n (\%) } \\
\hline Neurológico & 6 & $(50.0)$ & 3 & $(30.0)$ & 9 & $(40.9)$ \\
\hline Pulmonar & 5 & $(41.7)$ & 2 & $(20.0)$ & 7 & $(31.8)$ \\
\hline Cardiológico & 1 & $(8.3)$ & 2 & $(20.0)$ & 3 & (13.6) \\
\hline Post-RCP § & 0 & $(0.0)$ & 1 & $(10.0)$ & 1 & $(4.5)$ \\
\hline Postquirúrgico & 0 & $(0.0)$ & 1 & $(10.0)$ & 1 & $(4.5)$ \\
\hline Otro & 0 & $(0.0)$ & 1 & $(10.0)$ & 1 & $(4.5)$ \\
\hline \multicolumn{7}{|c|}{ Antecedentes, n (\%) } \\
\hline HTA II & 5 & $(41.7)$ & 5 & $(50.0)$ & 10 & $(45.5)$ \\
\hline EPOC ף & 4 & $(33.3)$ & 3 & $(30.0)$ & 7 & (31.8) \\
\hline Diabetes mellitus & 3 & $(25.0)$ & 4 & $(40.0)$ & 7 & $(31.8)$ \\
\hline $\begin{array}{l}\text { Enfermedad } \\
\text { cardiaca }\end{array}$ & & $(16.7)$ & 3 & $(30.0)$ & 5 & $(22.7)$ \\
\hline $\begin{array}{l}\text { Mortalidad } \\
\text { hospitalaria }\end{array}$ & & $(41.7)$ & 6 & $(60.0)$ & 11 & $(50.0)$ \\
\hline Mortalidad UCI & 3 & $(25.0)$ & 4 & $(40.0)$ & 7 & $(31.8)$ \\
\hline $\begin{array}{l}\text { Destete } \\
\text { ventilatorio }\end{array}$ & & $(50.0)$ & 5 & $(50.0)$ & 11 & $(50.0)$ \\
\hline $\begin{array}{l}\text { Antibióticos } \\
\text { previo }\end{array}$ & 11 & $(91.7)$ & 10 & $(100)$ & 21 & $(95.5)$ \\
\hline Traqueostomía & 10 & $(83.3)$ & 8 & $(80.0)$ & 18 & $(81.8)$ \\
\hline $\begin{array}{l}\text { Uso de } \\
\text { inotrópicos }\end{array}$ & & $(41.7)$ & 7 & $(70.0)$ & 12 & $(54.5)$ \\
\hline Transfusiones & & $(33.3)$ & 7 & $(70.0)$ & 11 & $(50.0)$ \\
\hline
\end{tabular}

* Evaluación Fisiológica Aguda y de Salud Crónica, † Expresados en mediana y desviación estándar, ₹ Score para Evaluación de Fallo Orgánico Secuencial, § Reanimación cardiopulmonar, || Hipertensión arterial, ๆ Enfermedad pulmonar obstructiva crónica. 
negativos, con solo un caso por gram positivo, el cual se presentó en TAV. El tipo de germen más comúnmente encontrado fue Klebsiella pneumoniae. Hubo una alta frecuencia de microrganismos multirresistentes en ambos tipos de infecciones. El resto de las características microbiológicas se muestran en la Tabla No 3.

\section{DISCUSION}

Este es el primer estudio sobre IRAV realizado en la institución. En la mayoría de las publicaciones se ha estudiado la NAV y muy pocos la TAV. Ambas patologías tuvieron una presencia similar a la informada en otros estudios, en caso de TAV entre 2.7 a $10.0 \%$ (7-11), mientras que para la NAV, fue semejante a los estudios realizados por Anselmo et al (12) y Dallas et al (8), no obstante puede variar la presencia hasta alcanzar el $28 \%$ (13), teniendo en cuenta el tipo de estudio.
La incidencia baja reportada en este estudio, puede estar relacionada, con la sola inclusión de pacientes que tenían cultivos positivos al momento del diagnóstico, en contraste con Nseir (11), quien realizó cultivos rutinarios al momento de la admisión a UCI y luego semanales al tener la sospecha clínica.

Hubo una mayor frecuencia de IRAV en personas de edad avanzada, con una proporción similar en mujeres y hombres, siendo los varones el $55 \%$ del total de la población con IRAV. Con respecto a esto, los estudios demuestran la mayor frecuencia en la tercera edad $(14,15)$, lo cual refleja el deterioro sistémico en este grupo etario (11). En cuanto al género, Rello et al (16), identificaron al sexo masculino, como un factor de riesgo independiente, asociado al desarrollo de la NAV.

Los diagnósticos predominantes fueron neurológicos y pulmonares. En el estudio de Iribarren et al (17), las patologías neurológicas fueron las que motivaron el ingreso a UCI, por su parte otros estudios, documentan las afecciones respiratorias como las principales causas de admisión a UCI en los pacientes con NAV y TAV (18). En este estudio la mayoría de los pacientes tenían APACHE-II, alrededor de 20, corroborándose con el estudio de Iribarren et al (17), donde se encontró que el puntaje de APACHE-II mayor o igual a 22 al momento del diagnóstico de NAV, se asoció a un incremento en la mortalidad.

Las comorbilidades que predominaron fueron hipertensión arterial, EPOC y diabetes mellitus, similar a lo informado en otros estudios $(8,18)$. La presencia de EPOC fue del $31.8 \%$ del total de infecciones. El EPOC constituye un factor de riesgo para la NAV (19) y estos pacientes son más propen- 
sos a tener colonización por gérmenes gram negativos en las vías respiratorias (11).

Hubo un caso de traqueobronquitis que evolucionó a NAV, resultado similar a lo encontrado por Abdo et al (12), donde no hubo caso de NAV en pacientes con TAV, aunque inferior con respecto a otras publicaciones (5), estando en desacuerdo con la teoría que la TAV es un estado intermedio para NAV (20). Diversos estudios demuestran que la administración de antibióticos en pacientes con TAV disminuye la incidencia de NAV (21), sin embargo esto puede ocurrir en presencia o no de terapia antibiótica (8), lo cual probablemente tenga relación con lo expuesto por Wunderink et al (22), donde la aparición de la TAV y la prevención de la NAV depende de la respuesta del huésped a nivel alveolar.

Se observaron comúnmente gérmenes gram negativos en las IRAV, predominando la Klebsiella pneumoniae y Pseudomona spp., tal como lo demuestran las publicaciones $(14,23)$, con poco porcentaje de Estafilococos aureus, similar a lo encontrado por otros artículos (23). La infección tardía se presentó en casi la totalidad de los pacientes, siendo mayor que la reportada $(8,23)$ y se ha asociado a alta tasa de gérmenes resistentes a drogas (24), como lo encontrado en el presente estudio, porcentaje alrededor del $70 \%$ en la NAV, coincidiendo con otras publicaciones $(5,8)$, lo cual podría estar relacionado a la administración de antibióticos previos (25). No obstante, el tratamiento antimicrobiano inicial, puede tener doble comportamiento, como factor protector para la NAV en los primeros días, así como selector de patógenos resistentes (26).

El número de días en UCI y de VM fueron mayores a dos semanas, similar a lo documentado por otros autores $(8,11,18)$. Las IRAV se han asociado a aumento en la estancia en UCI (2) y mayor duración de la ventilación mecánica $(4,16)$. Por su parte la mortalidad estuvo en el rango de los estudios previos $(11,18)$. La NAV incrementa dos veces el riesgo de fallecer (2), considerándose un factor independiente de mortalidad (27).

La necesidad de traqueostomía fue mayor que la reportada en Dallas et al (8), siendo en más de la mitad de los pacientes con traqueostomía tardía. Boles et al (28), reportaron menor incidencia de NAV en los pacientes con traqueostomía precoz, sin embargo un metaanálisis (29) encontró que la traqueostomía temprana no disminuye la NAV ni la mortalidad, pero si la duración de la VM y la estancia en UCI.

Alrededor de la mitad de los pacientes requirieron transfusión de hemoderivados; según lo publicado, las trasfusiones mayores a cuatro unidades se asocian al desarrollo de NAV (30). Un hallazgo interesante fue el alto porcentaje de pacientes con fracaso en el destete ventilatorio, ameritando el traslado a centro de unidad de cuidados de pacientes crónicos.

A pesar de las limitaciones propias del estudio retrospectivo y tener una muestra pequeña, los resultados fueron similares a lo publicado y pueden ser esos datos y proposiciones universales, adecuadas para considerar en instituciones regionales o locales. No obstante, se deben realizar nuevos estudios, analíticos y se deben implementar protocolos para prevenir IRAV y reducir la presencia de gérmenes resistentes.

\section{CONCLUSIÓN}

Se encontró incidencia baja de IRAV, con similar proporción de TAV y NAV. No obstante, fue elevada la presencia de gérmenes gram negativos, elevado porcentaje de microorganismos multirresistentes y alta tasa mortalidad.

CONFLICTOS DE INTERESES: ninguno que declarar.

FINANCIACIÓN: recursos propios de los autores

\section{REFERENCIAS BIBLIOGRÁFICAS}

1. Institute for Healthcare Improvement. 5 Million Lives Campaign. Getting Started Kit: Prevent Ventilator-Associated Pneumonia How-to Guide. Online 2008. Available from: URL: www.ihi.org.

2. Safdar N, Dezfulian C, Collard HR, Saint S. Clinical and economic consequences of ventilator- 
associated pneumonia: a systematic review. Crit Care Med. 2005;33(10):2184-93.

3. Restrepo MI, Anzueto A, Arroliga AC, Afessa B, Atkinson MJ, Ho NJ, et al. Economic burden of ventilator-associated pneumonia based on total resource utilization. Infect Control Hosp Epidemiol. 2010;31(5):509-15.

4. Koulenti D, Lisboa T, Brun-Buisson C, Krueger W, Macor A, Sole-Violan J, et al. Spectrum of practice in the diagnosis of nosocomial pneumonia in patients requiring mechanical ventilation in European intensive care units. Crit Care Med. 2009;37(8):2360-8.

5. Craven DE, Lei $Y$, Ruthazer R, Sarwar A, Hudcova J. Incidence and outcomes of ventilator-associated tracheobronchitis and pneumonia. Am J Med. 2013;126(6):542-9.

6. Bouza E, Pérez A, Muñoz P, Pérez M, Rincon C, Sánchez C, et al. Cardiovascular Infection Study Group: Ventilator-associated pneumonia after heart surgery: a prospective analysis and the value of surveillance. Crit Care Med. 2003;31(4):1964-1970.

7. Langer M, Cigada M, Mandelli M, Mosconi P, Tognoni G. Early onset pneumonia: a multicenter study in intensive care units. Intensive Care Med.1987;13(1):342-6.

8. Dallas J, Skrupky L, Abebe N, Boyle WA 3rd, Kollef MH. Ventilator associated tracheobronchitis in mixed surgical and medical ICU population. Chest. 2011;139(3):513-8.

9. Kampf G, Wischnewski N, Schulgen G, Schumacher M, Daschner F. Prevalence and risk factors for nosocomial lower respiratory tract infections in German hospitals. J Clin Epidemiol. 1998;5(2):495502.

10. Rello J, Ausina V, Castella J, Net A, Prats G. Nosocomial respiratory tract infections in multiple trauma patients. Chest. 1992;102(4):525-9.

11. Nseir S, Di Pompeo C, Pronnier P, Beague S, Onimus T, Saulnier F, et al. Nosocomial tracheobronchitis in mechanically ventilated patients: incidence, aetiology and outcome. Eur Respir $\mathrm{J}$. 2002;20(2):1483-9.

12. Abdo A, Castellanos R, González JC, Reyes R, Vázquez Y, Somoza ME, et al. Incidencia y consecuencias de la traqueobronquitis asociada a ventilación mecánica en unidades de cuidados intensivos. Rev cubana med. 2013;52:14-24.

13. Gupta A, Agrawal A, Mehrotra S, Singh A, Malik S, Khanna A. Incidence, risk stratification, antibiogram of pathogens isolated and clinical outcome of ventilator associated pneumonia. Indian J Crit Care Med. 2011;15:96-101.

14. Labaut Arévalo N, Riera Santiestebán R, Pérez Fuentes IA, Castañeda Carrazana Y. Neumonía asociada a la ventilación mecánica en una unidad de cuidados intensivos. MEDISAN. 2011;15:1759-64.

15. Callard HR, Saint S, Matthay MA. Prevention of ventilator-associated pneumonia: an evidencebased systematic review. Ann Intern Med. 2003;138:494-501.

16. Rello J, Ollendorf DA, Oster G, Vera-Llonch M, Bellm L, Redman R, et al. Epidemiology and outcomes of ventilator-associated pneumonia in a large US database. Chest;2002:122: 2115-21.

17. Iribarren $O$, Aranda J, Dorn L, Ferrada M, Ugarte H, Koscina $V$, et al. Factores de riesgo para mortalidad en neumonía asociada a ventilación mecánica. Rev Chil Infect. 2009;26:227-32.

18. Shahin J, Bielinski M, Guichon C, Flemming C, Kristof AS. Suspected ventilator- associated respiratory infection in severely ill patients: prospective observational study. Crit Care. 2013;17:1-7.

19. Torres A, Aznar R, Gattel JM, Jiménez P, González J, Ferrer A, et al. Incidence, risk, and prognosis factors of nosocomial pneumonia in mechanically ventilated patients. Am Rev Respir Dis. $1990 ; 142: 523-8$.

20. Craven DE, Chroneou A, Zias N, Hjalmarson KI. Ventilator-associated tracheobronchitis: the impact of targeted antibiotic therapy on patient outcomes. Chest. 2009;1352:521-8.

21. Nseir S, Favory R, Jozefowicz E, Decamps F, Dewavrin F, Brunin G, et al. Antimicrobial treatment for ventilator-associated tracheobronchitis: a randomized, controlled, multicenter study. Crit Care. 2008; 12:1-12.

22. Wunderink RG. Ventilator-Associated Tracheobronchitis. Public-Reporting Scam or Important Clinical Infection? Chest. 2011;139:485-8.

23. Castro M, Tartabull K, Nicolau E. Microorganismos aislados en pacientes con infecciones asociadas a la ventilación mecánica en los servicios de atención al grave. AMC. 2010;14:1-9.

24. Hospital-acquired pneumonia in adults: diagnosis, assessment of severity, initial antimicrobial therapy, and preventive strategies. A consensus statement, American Thoracic Society, November 1995. Am J Respir Crit Care Med. 1996;153:1711-25.

25. Trouillet JL, Chastre J, Vuagnat A, Joly-Guillou ML, Combaux D, Dombret MC, et al. Ventilatorassociated pneumonia caused by potentially drug-resistant bacteria. Am J Respir Crit Care Med. 1998;157:531-9.

26. Díaz O, Diaz E, Rello J. Factores de riesgo para el desarrollo de neumonía en pacientes intubados. Infect Dis Clin N Am. 2003;17:697-705.

27. Fagon JY, Chastre J, Vuagnat A, Trouillet JL, Novara A, Gibert C. Nosocomial pneumonia and mortality among patients in intensive care units. JAMA. 1996;275:866-9.

28. Boles JM, Bion J, Connors A, Herridge M, Marsh B, Melot C, et al. Weaning from mechanical ventilation. Eur Respir J. 2007;29:1033-5.

29. Griffiths J, Barber VS, Morgan L, Young JD. Systematic review and meta-analysis of studies of the timing of tracheostomy in adult patients undergoing artificial ventilation. BMJ. 2005;330:1243.

30. Leal SR, Rincón MD, García A, Herruzo A, Camacho P, Garnacho J, et al. Transfusion of blood components and postoperative infection in patients undergoing cardiac surgery. Chest. 2001;119:1461-8. 